\title{
Systems of conservation laws with third-order Hamiltonian structures
}

\author{
E.V. Ferapontov ${ }^{1}$, M.V. Pavlov ${ }^{2}$, R.F. Vitolo ${ }^{3}$ \\ ${ }^{1}$ Department of Mathematical Sciences, \\ Loughborough University, \\ Loughborough, Leics, UK \\ e.v.ferapontov@lboro.ac.uk \\ ${ }^{2}$ Lebedev Physical Institute of Russian Academy of Sciences, \\ Leninskij Prospekt 53, 119991 Moscow, Russia \\ mpavlov@itp.ac.ru \\ ${ }^{3}$ Department of Mathematics and Physics "E. De Giorgi", \\ University of Salento, Lecce, Italy \\ and Sezione INFN di Lecce \\ raffaele.vitolo@unisalento.it
}

\begin{abstract}
We investigate $n$-component systems of conservation laws that possess third-order Hamiltonian structures of differential-geometric type. The classification of such systems is reduced to the projective classification of linear congruences of lines in $\mathbb{P}^{n+2}$ satisfying additional geometric constraints. Algebraically, the problem can be reformulated as follows: for a vector space $W$ of dimension $n+2$, classify $n$-tuples of skew-symmetric 2-forms $A^{\alpha} \in \Lambda^{2}(W)$ such that

$$
\phi_{\beta \gamma} A^{\beta} \wedge A^{\gamma}=0,
$$

for some non-degenerate symmetric $\phi$.

MSC: 37K05, 37K10, 37K20, 37K25.

Keywords: System of Conservation Laws, Linear Congruence, Hamiltonian Operator, WDVV Equation, Projective Group, Reciprocal Transformation, Quadratic Complex, Monge Metric.
\end{abstract}


To Professor Boris Konopelchenko on the occasion of his 70th birthday

\section{Contents}

1 Introduction

1.1 Systems of conservation laws and line congruences in projective space . . . . . . . . . . . . . 3

1.2 Third-order Hamiltonian operators . . . . . . . . . . . 4

1.3 Hamiltonian systems of conservation laws . . . . . . . . 5

1.4 Examples . . . . . . . . . . . . . . . 7

1.5 Projective invariance . . . . . . . . . . . . . . . 10

1.6 Classification results . . . . . . . . . . . . . . . 11

1.7 Algebraic reformulation of the problem . . . . . . . . . 12

1.8 Symbolic computations . . . . . . . . . . . . . 12

2 Proofs 13

2.1 Conditions for a system to be Hamiltonian: proof of Theorem 113

2.2 Involutivity of system (11): proof of Theorem $2 \ldots \ldots$. . . 17

2.3 Integration of system (11): proof of Theorem $3 \ldots \ldots$. . . 20

2.4 Projective invariance: proof of Theorem $4 \ldots \ldots . \ldots 21$

2.5 Casimirs, Momentum, Hamiltonian . . . . . . . . . . 23

2.6 Algebraic reformulation of conditions (9), (10), (30) . . . . 25

2.7 Classification results: proof of Theorems $5,6 \ldots \ldots 25$

3 Concluding remarks $\quad 27$

4 Acknowledgements $\quad 28$ 


\section{Introduction}

\subsection{Systems of conservation laws and line congruences in projective space}

Systems of conservation laws are $n$-component first-order PDEs of the form

$$
u_{t}^{i}=\left(V^{i}(\mathbf{u})\right)_{x},
$$

$i=1, \ldots, n$, where $V^{i}(\mathbf{u})$ is a (nonlinear) vector of fluxes. We will assume that the characteristic speeds of system (1), that is, the eigenvalues of the Jacobian matrix of the fluxes $V^{i}$, are real and distinct (condition of strict hyperbolicity). Systems of conservation laws appear in a wide range of applications in continuum mechanics and mathematical physics, see e.g. $[21,25,35,32,34,33]$. Following the geometric approach of [1, 2], with system (1) we associate a congruence (that is, $n$-parameter family of lines),

$$
y^{i}=u^{i} y^{n+1}+V^{i} y^{n+2},
$$

in auxiliary projective space $\mathbb{P}^{n+1}$ with homogeneous coordinates $\left(y^{1}: \cdots\right.$ : $\left.y^{n+2}\right)$. It was demonstrated in $[1,2]$ that various standard concepts of the theory of conservation laws such as rarefaction curves, shock curves, linear degeneracy, reciprocal transformations, etc, acquire a simple interpretation in the language of the projective theory of congruences. In particular, reciprocally related systems (1) correspond to projectively equivalent congruences (2). Algebro-geometric aspects of this correspondence were investigated in $[6,7,8,26]$.

In this paper we utilise the above geometric correspondence for the classification of systems (1) possessing third-order Hamiltonian structures. We will show that congruences associated with Hamiltonian systems are necessarily linear, that is, they are specified by $n$ linear relations among the Plücker coordinates (in geometric language, codimension $n$ linear sections of the Grassmannian $G(1, n+1)$ ). We recall that the lines of a linear congruence in $\mathbb{P}^{n+1}$ can be characterised geometrically as $n$-secants of the focal variety (jump locus), which is a codimension two subvariety in $\mathbb{P}^{n+1}$ (possibly, reducible):

- For $n=2$ the focal variety of a linear congruence consists of 2 skew lines in $\mathbb{P}^{3}$.

- For $n=3$ the focal variety of a generic linear congruence is a projection of the Veronese surface $V^{2} \subset \mathbb{P}^{5}$ into $\mathbb{P}^{4}[5]$. 
- For $n=4$ the focal variety is a Palatini threefold in $\mathbb{P}^{5}$ [28], etc.

In parametrisation (2), the Plücker coordinates are just $u^{i}, V^{i}, u^{i} V^{j}-u^{j} V^{i}$. Imposing $n$ linear relations among the Plücker coordinates we obtain a linear system for the fluxes $V^{i}$ which implies that $V^{i}$ are rational in $u$. Systems associated with linear congruences are linearly degenerate, and satisfy the Temple property [2].

\subsection{Third-order Hamiltonian operators}

Third-order Hamiltonian operators of differential-geometric type were introduced by Dubrovin and Novikov in [10], and subsequently investigated in $[31,9,30,27,4,15,16]$. They are defined by the general formula

$$
\begin{aligned}
P^{i j}=g^{i j} \partial_{x}^{3}+b_{k}^{i j} u_{x}^{k} \partial_{x}^{2}+\left(c_{k}^{i j} u_{x x}^{k}\right. & \left.+c_{k m}^{i j} u_{x}^{k} u_{x}^{m}\right) \partial_{x} \\
& +d_{k}^{i j} u_{x x x}^{k}+d_{k m}^{i j} u_{x x}^{k} u_{x}^{m}+d_{k m n}^{i j} u_{x}^{k} u_{x}^{m} u_{x}^{n},
\end{aligned}
$$

where $u^{i}, i=1, \ldots, n$, are the dependent variables, and the coefficients $g^{i j}, \ldots, d_{k m n}^{i j}$ are functions of $u^{i}$ only; $\partial_{x}$ stands for the total derivative with respect to $x$. The requirement that the corresponding Poisson bracket,

$$
\{F, G\}=\int \frac{\delta F}{\delta u^{i}} P^{i j} \frac{\delta G}{\delta u^{j}} d x,
$$

is skew-symmetric and satisfies the Jacobi identities, imposes strong constraints on the coefficients of $P$. We restrict our considerations to the nondegenerate case, $\operatorname{det} g^{i j} \neq 0$; in what follows we use $g^{i j}$ for raising and lowering indices. It was demonstrated in $[31,9]$ that there exists a coordinate system (flat coordinates) in which Hamiltonian operator $P$ takes a simple factorised form [27],

$$
P^{i j}=\partial_{x}\left(g^{i j} \partial_{x}+c_{k}^{i j} u_{x}^{k}\right) \partial_{x} .
$$

In what follows we will always work in the flat coordinates, and keep for them the notation $u^{i}$; note that $u^{i}$ are nothing but the densities of Casimirs of the corresponding Hamiltonian operator. Introducing $c_{i j k}=g_{i q} g_{j p} c_{k}^{p q}$ one can show [30] that the skew-symmetry conditions and the Jacobi identities for operator (3) are equivalent to

$$
\begin{gathered}
g_{m n, k}=-c_{m n k}-c_{n m k}, \\
c_{m n k}=-c_{m k n}, \\
c_{m n k}+c_{n k m}+c_{k m n}=0, \\
c_{m n k, l}=-g^{p q} c_{p m l} c_{q n k} .
\end{gathered}
$$


Equations (4a)-(4c) imply [15]

$$
c_{s k m}=\frac{1}{3}\left(g_{s m, k}-g_{s k, m}\right) .
$$

The elimination of $c$ from equations (4) gives a system for the metric $g$,

$$
\begin{gathered}
g_{m k, n}+g_{k n, m}+g_{m n, k}=0, \\
g_{m[k, n] l}=-\frac{1}{3} g^{p q} g_{p[l, m]} g_{q[k, n]} .
\end{gathered}
$$

Equations (6a) mean that $g$ is a Monge metric, and as such is an object of projective differential geometry. Building on the correspondence of Monge metrics to quadratic complexes of lines in $\mathbb{P}^{n}$, in $[15,16]$ we proposed a classification of Hamiltonian operators (3) for $n \leq 4$.

In what follows we will also need the result of Balandin and Potemin [4] according to which the general solution of system (6) is given by the formula

$$
g_{i j}=\phi_{\beta \gamma} \psi_{i}^{\beta} \psi_{j}^{\gamma},
$$

where $\phi_{\beta \gamma}$ is a non-degenerate constant symmetric matrix, and

$$
\psi_{k}^{\gamma}=\psi_{k m}^{\gamma} u^{m}+\omega_{k}^{\gamma}
$$

here $\psi_{k m}^{\gamma}$ and $\omega_{k}^{\gamma}$ are constants such that $\psi_{k m}^{\gamma}=-\psi_{m k}^{\gamma}$. These constants have to satisfy an additional set of quadratic relations,

$$
\begin{gathered}
\phi_{\beta \gamma}\left(\psi_{i s}^{\beta} \psi_{j k}^{\gamma}+\psi_{j s}^{\beta} \psi_{k i}^{\gamma}+\psi_{k s}^{\beta} \psi_{i j}^{\gamma}\right)=0, \\
\phi_{\beta \gamma}\left(\omega_{i}^{\beta} \psi_{j k}^{\gamma}+\omega_{j}^{\beta} \psi_{k i}^{\gamma}+\omega_{k}^{\beta} \psi_{i j}^{\gamma}\right)=0,
\end{gathered}
$$

whose algebraic meaning was clarified in [16]. An important invariant of Hamiltonian operator (3) is its singular variety, $\operatorname{det} g_{i j}=0$, which, due to (7), is a double hypersurface of degree $n-1$ :

$$
\operatorname{det} g=\operatorname{det} \phi(\operatorname{det} \psi)^{2} ;
$$

here the degree of $\operatorname{det} \psi$ equals $n-1[16]$.

\subsection{Hamiltonian systems of conservation laws}

In this paper we are interested in Hamiltonian systems of conservation laws, namely systems (1) possessing third-order Hamiltonian structures (3):

$$
\mathbf{u}_{t}=(V(\mathbf{u}))_{x}=P \frac{\delta H}{\delta \mathbf{u}}
$$


for some (nonlocal) Hamiltonian functionals $H$. Examples of such systems include Monge-Ampère equations, as well as various versions of WDVV equations, see [11] for their geometric treatment based on the theory of Frobenius manifolds. Our main results in this direction can be summarised as follows.

Theorem 1. The necessary and sufficient conditions for a conservative system (1) to possess third-order Hamiltonian operator (3) are the following:

$$
\begin{aligned}
& g_{i m} V_{j}^{m}=g_{j m} V_{i}^{m}, \\
& c_{m k l} V_{i}^{m}+c_{m i k} V_{l}^{m}+c_{m l i} V_{k}^{m}=0, \\
& V_{i j}^{k}=g^{k s} c_{s m j} V_{i}^{m}+g^{k s} c_{s m i} V_{j}^{m},
\end{aligned}
$$

here lower indices of $V^{m}$ denote partial derivatives: $V_{i}^{m}=\partial V^{m} / \partial u^{i}$, etc.

In Theorem 7 of Section 2.5 we present explicit formulae for the corresponding Casimirs, Momentum and Hamiltonian. The proof of Theorem 1 can be found in Section 2.1.

Conditions (11) are analogous to Tsarev's conditions in the theory of first-order homogeneous Hamiltonian operators [36]. System (11) possesses a number of important properties, in particular, in Section 2.2 we establish the following result:

Theorem 2. System (11) is in involution. Its general solution depends on $\leq \frac{n(n+3)}{2}$ arbitrary constants.

It is quite remarkable that system (11), which is a linear involutive system with non-constant coefficients, can be integrated in closed form (Section 2.3). This leads to the following result (Section 2.3):

Theorem 3. For Hamiltonian system (1) the following conditions hold:

- The associated congruence (2) is linear.

- System (1) is linearly degenerate and belongs to the Temple class.

- The fluxes $V^{i}$ are rational functions of the form

$$
V^{i}=\frac{Q^{i}}{\operatorname{det} \psi},
$$

where det $\psi$ is a polynomial of degree $n-1$ defining the singular variety, and $Q^{i}$ are polynomials of degree $n$. 
Note that for $n \geq 4$ systems of conservation laws possessing third-order Hamiltonian structures are neither diagonalisable nor integrable in general.

Based on the classification of linear congruences in $\mathbb{P}^{3}$ and $\mathbb{P}^{4}$ dating back to the classical work of Castelnuovo [5], this leads to a complete description of Hamiltonian systems (1) for $n=2,3$, see Section 1.6.

\subsection{Examples}

Here we list examples of conservative systems (1) with third-order Hamiltonian structures (3) that will feature in the classification results below. In order to simplify the expressions for the Hamiltonian densities we introduce potential coordinates $b^{i}$ via $u^{i}=b_{x}^{i}$. In these coordinates system (1) will no longer be quasilinear, and third-order Hamiltonian operator (3) takes a first-order form, see (21), (22).

Example 1. A linear $n$-component system of conservation laws,

$$
u_{t}^{i}=\left(a_{j}^{i} u^{j}\right)_{x}
$$

$a_{j}^{i}=$ const, possesses third-order Hamiltonian formulation

$$
u_{t}^{i}=\eta^{i j} \partial_{x}^{3} \frac{\delta H}{\delta u^{j}},
$$

$\eta^{i j}=$ const, with the nonlocal Hamiltonian

$$
H=-\frac{1}{2} \int \eta_{j p} a_{k}^{p} b^{j} b^{k} d x .
$$

In this case, conditions (11) reduce to $\eta_{j p} a_{k}^{p}=\eta_{k p} a_{j}^{p}$, which means that the operator $a$ is symmetric with respect to the metric $\eta$.

The associated congruence (2) is the set of lines that intersect $n$ linear subspaces of codimension two in $\mathbb{P}^{n+1}$ (the union of these subspaces constitutes the focal variety). These subspaces can be described explicitly: let $\lambda^{k}$ be the eigenvalues of $a$ with the corresponding left eigenvectors $\xi^{k}$, that is, $a_{i}^{j} \xi_{j}^{k}=\lambda^{k} \xi_{i}^{k}$. Then the $k$-th focal subspace is defined by two linear equations, $y^{0}=\lambda^{k}, \xi_{j}^{k} y^{j}=0$.

Example 2. The simplest WDVV equation [11], $f_{t t t}=f_{x x t}^{2}-f_{x x x} f_{x t t}$, can be reduced to a 3 -component conservative form,

$$
u_{t}^{1}=u_{x}^{2}, \quad u_{t}^{2}=u_{x}^{3}, \quad u_{t}^{3}=\left(\left(u^{2}\right)^{2}-u^{1} u^{3}\right)_{x},
$$


by setting $u^{1}=f_{x x x}, u^{2}=f_{x x t}, u^{3}=f_{x t t}$. System (12) possesses a Hamiltonian formulation $u_{t}=P \delta H / \delta u$ [13], with the homogeneous third-order Hamiltonian operator

$$
P=\partial_{x}\left(\begin{array}{ccc}
0 & 0 & \partial_{x} \\
0 & \partial_{x} & -\partial_{x} u^{1} \\
\partial_{x} & -u^{1} \partial_{x} & \partial_{x} u^{2}+u^{2} \partial_{x}+u^{1} \partial_{x} u^{1}
\end{array}\right) \partial_{x}
$$

and the nonlocal Hamiltonian

$$
\left.H=-\int\left(\frac{1}{2} u^{1}\left(b^{2}\right)^{2}+b^{2} b^{3}\right)\right) d x
$$

Note that system (12) possesses a compatible first-order Hamiltonian formulation, as well as a Lax pair, which elucidate its integrability [13].

The associated congruence (2) was thoroughly investigated in [2]. It consists of trisecant lines of the focal variety which, in this case, is a generic projection of the Veronese surface $V^{2} \subset \mathbb{P}^{5}$ into $\mathbb{P}^{4}$. Various generalisations of this example can be found in [19, 20, 29, 23].

Example 3. The following 4-component conservative system was obtained in [3] in the classification of non-diagonalisable linearly degenerate systems of Temple's class whose characteristic speeds are harmonic (have cross-ratio equal to -1$)$ :

$$
\begin{aligned}
& u_{t}^{1}=u_{x}^{3}, \\
& u_{t}^{2}=u_{x}^{4}, \\
& u_{t}^{3}=\left(\frac{u^{1} u^{2} u^{4}+u^{3}\left(\left(u^{3}\right)^{2}+\left(u^{4}\right)^{2}-\left(u^{2}\right)^{2}-1\right)}{u^{1} u^{3}+u^{2} u^{4}}\right)_{x}, \\
& u_{t}^{4}=\left(\frac{u^{1} u^{2} u^{3}+u^{4}\left(\left(u^{3}\right)^{2}+\left(u^{4}\right)^{2}-\left(u^{1}\right)^{2}-1\right)}{u^{1} u^{3}+u^{2} u^{4}}\right)_{x} .
\end{aligned}
$$

System (13) possesses a Hamiltonian representation $u_{t}=P \delta H / \delta u$ where the third-order Hamiltonian operator $P$ is generated by the Monge metric

$$
g_{i j}=\left(\begin{array}{cccc}
\left(u^{2}\right)^{2}+\left(u^{3}\right)^{2}+1 & -u^{1} u^{2}+u^{3} u^{4} & -u^{1} u^{3}+u^{2} u^{4} & -2 u^{2} u^{3} \\
-u^{1} u^{2}+u^{3} u^{4} & \left(u^{1}\right)^{2}+\left(u^{4}\right)^{2}+1 & -2 u^{1} u^{4} & u^{1} u^{3}-u^{2} u^{4} \\
-u^{1} u^{3}+u^{2} u^{4} & -2 u^{1} u^{4} & \left(u^{1}\right)^{2}+\left(u^{4}\right)^{2} & u^{1} u^{2}-u^{3} u^{4} \\
-2 u^{2} u^{3} & u^{1} u^{3}-u^{2} u^{4} & u^{1} u^{2}-u^{3} u^{4} & \left(u^{2}\right)^{2}+\left(u^{3}\right)^{2}
\end{array}\right) .
$$


Due to $\operatorname{det} g=\left(u^{1} u^{3}+u^{2} u^{4}\right)^{2}$, its singular variety consists of a double quadric and a double plane an infinity. The corresponding nonlocal Hamiltonian $H$ is given by

$$
H=-\frac{1}{2} \int\left(b^{1} b^{3}+b^{2} b^{4}+x\left(b^{1} u^{3}-u^{1} b^{3}+b^{2} u^{4}-u^{2} b^{4}\right)\right) d x,
$$

note the explicit dependence on $x$. Integrability of system (13) can be demonstrated as follows. Introducing the $2 \times 3$ matrix

$$
Z=\left(\begin{array}{lll}
u^{1} & u^{2} & 1 \\
u^{3} & u^{4} & 0
\end{array}\right)
$$

one can represent (13) in matrix form (compare with Sect. 4 in [17]),

$$
Z_{t}=\left(a Z Z^{T} Z+b Z\right)_{x}
$$

where $a=\frac{1}{u^{1} u^{3}+u^{2} u^{4}}, \quad b=-\frac{\left(u^{1}\right)^{2}+\left(u^{2}\right)^{2}+1}{u^{1} u^{3}+u^{2} u^{4}}$. Introducing the $5 \times 5$ skewsymmetric matrix

$$
S=\left(\begin{array}{cc}
0 & Z \\
-Z^{t} & 0
\end{array}\right)
$$

one can rewrite (14) as a matrix Hopf-type equation,

$$
S_{t}=\left(b S-a S^{3}\right)_{x},
$$

with the Lax pair

$$
\psi_{x}=\lambda S \psi, \quad \psi_{t}=\lambda\left(b S-a S^{3}\right) \psi
$$

Congruence (2) associated with system (13) is related to the Cartan isoparametric hypersurface in $S^{5}$, see [3] for further details. Note that its focal variety is reducible.

Example 4. Let us consider a class of conservative 4-component systems of the form

$$
u_{t}^{1}=u_{x}^{2}, \quad u_{t}^{2}=u_{x}^{3}, \quad u_{t}^{3}=u_{x}^{4}, \quad u_{t}^{4}=\left[f\left(u^{1}, \ldots, u^{4}\right)\right]_{x} .
$$

Under the substitution $u^{1}=f_{x x x x}, u^{2}=f_{x x x t}, u^{3}=f_{x x t t}, u^{4}=f_{x t t t}$ they reduce to a scalar fourth-order PDE for $f(x, t)$. One can show that modulo equivalence transformations there exist only two types of such systems possessing third-order Hamiltonian structures: 
Case 1. $f=\left(u^{2}\right)^{2}-u^{1} u^{3}$. The corresponding system possesses a Hamiltonian formulation $u_{t}=P \delta H / \delta u$ with the third-order Hamiltonian operator

$$
P=\partial_{x}\left(\begin{array}{cccc}
0 & 0 & 0 & \partial_{x} \\
0 & 0 & \partial_{x} & 0 \\
0 & \partial_{x} & 0 & -\partial_{x} u^{1} \\
\partial_{x} & 0 & -u^{1} \partial_{x} & \partial_{x} u^{2}+u^{2} \partial_{x}
\end{array}\right) \partial_{x}
$$

and the nonlocal Hamiltonian

$$
H=-\frac{1}{2} \int\left(u^{1}\left(b^{2}\right)^{2}+2 b^{2} b^{4}+\left(b^{3}\right)^{2}\right) d x .
$$

Case 2. $f=\left(u^{3}\right)^{2}-u^{2} u^{4}+u^{1}$. The corresponding system possesses a Hamiltonian formulation $u_{t}=P \delta H / \delta u$ with the third-order Hamiltonian operator

$$
P=\partial_{x}\left(\begin{array}{cccc}
\partial_{x} & 0 & 0 & 0 \\
0 & 0 & 0 & \partial_{x} \\
0 & 0 & \partial_{x} & -\partial_{x} u^{2} \\
0 & \partial_{x} & -u^{2} \partial_{x} & \partial_{x} g+g \partial_{x}
\end{array}\right) \partial_{x}
$$

where $g=u^{3}+\frac{1}{2}\left(u^{2}\right)^{2}$, and the nonlocal Hamiltonian

$$
H=\int\left(b^{2} b^{3} u^{3}-b^{1} b^{2}-b^{3} b^{4}\right) d x .
$$

We point out that systems from cases 1, 2 are likely to be non-integrable.

\subsection{Projective invariance}

The class of conservative systems (1) is invariant under reciprocal transformations of the form

$$
\begin{aligned}
d \tilde{x} & =\left(a_{i} u^{i}+a\right) d x+\left(a_{i} V^{i}+b\right) d t, \\
d \tilde{t} & =\left(b_{i} u^{i}+c\right) d x+\left(b_{i} V^{i}+d\right) d t,
\end{aligned}
$$

which can be viewed as nonlocal changes of the independent variables $x, t$; here $a_{i}, b_{i}, a, b, c, d$ are arbitrary constants. It was shown in $[1,2]$ that, along with affine transformations of the dependent variables $u^{i}$, reciprocal transformations generate the group $S L(n+2)$ which acts by projective transformations on the associated congruence (2). It is remarkable that these transformations preserve the Hamiltonian property. 
Theorem 4. The class of conservative systems (1) possessing third-order Hamiltonian formulation (3) is invariant under reciprocal transformations (15).

We prove this result in Section 2.4. Note that, in contrast to third-order operators (3), first-order Hamiltonian structures of Dubrovin-Novikov type are not reciprocally invariant, and generally become nonlocal [14].

Remark. In $[15,16]$ we have classified third-order Hamiltonian operators/systems modulo the restricted group of reciprocal transformations that change the independent variable $x$ only,

$$
d \tilde{x}=\left(a_{i} u^{i}+a\right) d x+\left(a_{i} V^{i}+b\right) d t, \quad d \tilde{t}=d t .
$$

In the 3-component case this resulted in the 5 canonical forms. Modulo extended transformations (15), all of them are equivalent to that of Example 2 from Section 1.4.

Ultimately, the classification of Hamiltonian systems of conservation laws (1) up to reciprocal transformations (15) reduces to projective classification of the associated congruences (2).

\subsection{Classification results}

Here we summarise the classification results of Hamiltonian systems of conservation laws with $n=2$ and 3 components. The classification is performed modulo reciprocal/projective transformations as discussed in Section 1.5. We always assume that system (1) is strictly hyperbolic, and that the metric $g$ defining Hamiltonian operator (3) is non-degenerate.

The existing classification of linear congruences in $\mathbb{P}^{3}$ and $\mathbb{P}^{4}$ readily leads to the classification of 2- and 3-component Hamiltonian systems of conservation laws. Thus, every linear congruence in $\mathbb{P}^{3}$ consists of bisecants of two skew lines in $\mathbb{P}^{3}$. This leads to

Theorem 5. For $n=2$, every Hamiltonian system of conservation laws is linearisable (that is, equivalent to 2-component case of Example 1 from Section 1.4).

Linear congruences in $\mathbb{P}^{4}$ were classified by Castelnuovo in [5]: they can be obtained as trisecant lines of suitable projections of the Veronese surface from $\mathbb{P}^{5}$ into $\mathbb{P}^{4}$. Thus, all generic linear congruences are projectively equivalent (non-generic projections correspond to systems with degenerate Hamiltonian operators). 
Theorem 6. For $n=3$, every Hamiltonian system of conservation laws is either linearisable (that is, equivalent to 3-component case of Example 1 from Section 1.4), or equivalent to the system of WDVV equations (Example 2 from Section 1.4).

Theorems 5, 6 are proved in Section 2.7. It follows that all 3-component systems of conservation laws with third-order Hamiltonian structures are automatically integrable.

The case $n=4$ is far more complicated, primarily, due to the fact that there exists no classification of linear congruences in $\mathbb{P}^{5}$. Only partial results are currently available. In particular, 4-component Hamiltonian systems (1) associated with third-order Hamiltonian operators are not integrable in general.

\subsection{Algebraic reformulation of the problem}

Linear congruences in $\mathbb{P}^{n+1}$ are defined by $n$ linear relations in the Plücker coordinates. Setting $\mathbb{P}^{n+1}=\mathbb{P}(W)$ where $W$ is a vector space of dimension $n+2$, these linear relations correspond to the choice of an $n$-dimensional subspace $A \subset \Lambda^{2}(W)$. Let $A^{1}, \ldots, A^{n}$ denote a basis of $A$. The condition that the corresponding system (1) is Hamiltonian, is equivalent to the existence of a non-degenerate symmetric matrix $\phi_{\beta \gamma}$, the same as in (7), such that

$$
\phi_{\beta \gamma} A^{\beta} \wedge A^{\gamma}=0,
$$

see Section 2.6. The existence of such relation does not depend on the choice of basis, and imposes strong constraints on $A$. Despite its apparent simplicity, the classification of normal forms of such subspaces is an open problem (starting with $n=4$ ).

\subsection{Symbolic computations}

Symbolic computations were performed by CDE [38], a Reduce [37] package for integrability of PDEs. CDE (by one of us, RFV) can compute: Fréchet derivatives, formal adjoints, symmetries and conservation laws, Hamiltonian operators, and their brackets. Examples are available from [38], and a User's manual is included in the official Reduce manual; a book with numerous detailed computations is to appear soon [24]. 


\section{Proofs}

\subsection{Conditions for a system to be Hamiltonian: proof of Theorem 1}

In this section we derive the necessary and sufficient conditions for system (1) to possesses Hamiltonian structure (3).

Theorem 1. The necessary and sufficient conditions for a conservative system (1) to possess third-order Hamiltonian operator (3) are the following:

$$
\begin{aligned}
& g_{i m} V_{j}^{m}=g_{j m} V_{i}^{m}, \\
& c_{m k l} V_{i}^{m}+c_{m i k} V_{l}^{m}+c_{m l i} V_{k}^{m}=0, \\
& V_{i j}^{k}=g^{k s} c_{s m j} V_{i}^{m}+g^{k s} c_{s m i} V_{j}^{m},
\end{aligned}
$$

here low indices of $V^{m}$ denote partial derivatives, $V_{i}^{m}=\partial V^{m} / \partial u^{i}$, etc.

Proof. The proof is based on the Kersten-Krasil'shchik-Verbovetsky approach to Hamiltonian operators [22] which can be summarised as follows. Consider an evolutionary system of the form

$$
F^{i}=u_{t}^{i}-f^{i}\left(t, x, u, u_{x}, u_{x x}, \ldots\right)=0,
$$

with the formal linearization (Fréchet derivative) $\ell_{F}$. Let $P$ be a Hamiltonian operator, that is, a skew-adjoint operator with zero Schouten bracket, $[P, P]=0$. If system (17) possesses $P$ as a Hamiltonian structure, then $P$ maps variational derivatives of conserved densities of (17) into generalized (higher) symmetries, that is,

$$
\ell_{F} \circ P=P^{*} \circ \ell_{F}^{*}
$$

Let us introduce the adjoint system (cotangent covering) of system (17),

$$
\left\{\begin{array}{l}
F=0 \\
\ell_{F}^{*}(p)=0
\end{array}\right.
$$

where $p$ is an auxiliary (vector) variable. Then (18) is equivalent to

$$
\ell_{F}(P(p))=0,
$$

which must hold identically modulo (19). Note that the idea of representing Hamiltonian operators $P$ by linear differential expressions of type $P(p)$ 
was used in [18] to compute Hamiltonian cohomology. The advantage of the above formulation is that finding Hamiltonian operators amounts to solving a problem which is computationally the same as finding generalized symmetries.

To apply this technique to system (1) we introduce a potential substitution $u^{i}=b_{x}^{i}$, for reasons that will become clear soon, obtaining a nonquasilinear system

$$
b_{t}^{i}=V^{i}\left(\mathbf{b}_{x}\right)
$$

This substitution turns Hamiltonian operator (3) into a first-order operator,

$$
P^{i j}=-\left(g^{i j}\left(\mathbf{b}_{x}\right) \partial_{x}+c_{k}^{i j}\left(\mathbf{b}_{x}\right) b_{x x}^{k}\right),
$$

and the corresponding Hamiltonian can be calculated explicitly (see Section 2.5). Note that the above Hamiltonian operator is not of DubrovinNovikov type as its coefficients $g^{i s}\left(\mathbf{b}_{x}\right)$ and $c_{k}^{i s}\left(\mathbf{b}_{x}\right)$ loose their geometric interpretation: their transformation rule is no longer tensorial. The linearisation operator of system (21) is

$$
\ell_{F}(\boldsymbol{\varphi})=D_{t} \varphi^{i}-\frac{\partial V^{i}}{\partial b_{x}^{j}} D_{x} \varphi^{j}
$$

with the adjoint

$$
\ell_{F}^{*}(\boldsymbol{\psi})=-D_{t} \psi_{k}+D_{x}\left(\frac{\partial V^{i}}{\partial b_{x}^{k}} \psi_{i}\right) .
$$

The adjoint system is

$$
\begin{aligned}
& b_{t}^{i}=V^{i}\left(\mathbf{b}_{x}\right), \\
& p_{k, t}=\frac{\partial^{2} V^{i}}{\partial b_{x}^{k} \partial b_{x}^{h}} b_{x x}^{h} p_{i}+\frac{\partial V^{i}}{\partial b_{x}^{k}} p_{i, x} .
\end{aligned}
$$

Setting $P(\mathbf{p})=-g^{i j} p_{j, x}-c_{k}^{i j} b_{x x}^{k} p_{j}$, condition (20) takes the form

$$
\begin{gathered}
\ell_{F}(P(\mathbf{p}))=-\frac{\partial g^{i j}}{\partial b_{x}^{k}} b_{x t}^{k} p_{j, x}-g^{i j} p_{j, x t}-\frac{\partial c_{k}^{i j}}{\partial b_{x}^{h}} b_{x t}^{h} b_{x x}^{k} p_{j}-c_{k}^{i j} b_{x x t}^{k} p_{j}-c_{k}^{i j} b_{x x}^{k} p_{j, t} \\
+\frac{\partial V^{i}}{\partial b_{x}^{j}}\left(\frac{\partial g^{j k}}{\partial b_{x}^{h}} b_{x x}^{h} p_{k, x}+g^{j k} p_{k, x x}+\frac{\partial c_{k}^{j h}}{\partial b_{x}^{l}} b_{x x}^{l} b_{x x}^{k} p_{h}+c_{k}^{j h} b_{x x x}^{k} p_{h}+c_{k}^{j h} b_{x x}^{k} p_{h, x}\right) .
\end{gathered}
$$

Using differential consequences of the adjoint system,

$$
b_{t x}^{i}=V_{x}^{i}, \quad b_{t x x}^{i}=V_{x x}^{i}, \quad p_{k, t x}=D_{x x} \frac{\partial V^{i}}{\partial b_{x}^{k}} p_{i}+2 D_{x} \frac{\partial V^{i}}{\partial b_{x}^{k}} p_{i, x}+\frac{\partial V^{i}}{\partial b_{x}^{k}} p_{i, x x},
$$


we obtain

$$
\begin{aligned}
\ell_{F} & P(\mathbf{p}))=\left(-g^{i j} \frac{\partial V^{h}}{\partial b_{x}^{j}}+\frac{\partial V^{i}}{\partial b_{x}^{j}} g^{j h}\right) p_{h, x x} \\
+ & \left(-\frac{\partial g^{i h}}{\partial b_{x}^{k}} V_{x}^{k}-g^{i j} 2 D_{x} \frac{\partial V^{h}}{\partial b_{x}^{j}}-c_{k}^{i j} b_{x x}^{k} \frac{\partial V^{h}}{\partial b_{x}^{j}}\right. \\
& \left.+\frac{\partial V^{i}}{\partial b_{x}^{j}} \frac{\partial g^{j h}}{\partial b_{x}^{k}} b_{x x}^{k}+\frac{\partial V^{i}}{\partial b_{x}^{j}} c_{k}^{j h} b_{x x}^{k}\right) p_{h, x} \\
+ & \left(-g^{i j} D_{x x} \frac{\partial V^{h}}{\partial b_{x}^{j}}-\frac{\partial c_{k}^{i h}}{\partial b_{x}^{j}} V_{x}^{j} b_{x x}^{k}-c_{k}^{i h} V_{x x}^{k}-c_{k}^{i j} b_{x x}^{k} \frac{\partial^{2} V^{h}}{\partial b_{x}^{j} \partial b_{x}^{l}} b_{x x}^{l}\right. \\
+ & \left.\frac{\partial V^{i}}{\partial b_{x}^{j}}\left(\frac{\partial c_{k}^{j h}}{\partial b_{x}^{l}} b_{x x}^{l} b_{x x}^{k}+c_{k}^{j h} b_{x x x}^{k}\right)\right) p_{h} .
\end{aligned}
$$

The above expression is linear in $p_{h}, p_{h, x}, p_{h, x x}$, and the coefficients are polynomials in $b_{x x}^{i}, b_{x x x}^{i}$. So, the expression vanishes if and only if

$$
\begin{aligned}
& -g^{i j} \frac{\partial V^{h}}{\partial b_{x}^{j}}+\frac{\partial V^{i}}{\partial b_{x}^{j}} g^{j h}=0 \\
& -g^{i k} \frac{\partial^{2} V^{h}}{\partial b_{x}^{k} \partial b_{x}^{l}}-c_{k}^{i h} \frac{\partial V^{k}}{\partial b_{x}^{l}}+\frac{\partial V^{i}}{\partial b_{x}^{k}} c_{l}^{k h}=0 \\
& -\frac{\partial g^{i h}}{\partial b_{x}^{k}} \frac{\partial V^{k}}{\partial b_{x}^{l}}-g^{i j} 2 \frac{\partial^{2} V^{h}}{\partial b_{x}^{j} \partial b_{x}^{l}}-c_{l}^{i j} \frac{\partial V^{h}}{\partial b_{x}^{j}}+\frac{\partial V^{i}}{\partial b_{x}^{j}} \frac{\partial g^{j h}}{\partial b_{x}^{l}}+\frac{\partial V^{i}}{\partial b_{x}^{j}} c_{l}^{j h}=0 \\
& -g^{i j} \frac{\partial^{3} V^{h}}{\partial b_{x}^{j} \partial b_{x}^{l} \partial b_{x}^{m}}-\frac{1}{2}\left(\frac{\partial c_{m}^{i h}}{\partial b_{x}^{j}} \frac{\partial V^{j}}{\partial b_{x}^{l}}+\frac{\partial c_{l}^{i h}}{\partial b_{x}^{j}} \frac{\partial V^{j}}{\partial b_{x}^{m}}\right)-c_{k}^{i h} \frac{\partial^{2} V^{k}}{\partial b_{x}^{l} b_{x}^{m}} \\
& -\frac{1}{2}\left(c_{m}^{i j} \frac{\partial^{2} V^{h}}{\partial b_{x}^{j} \partial b_{x}^{l}}+c_{l}^{i j} \frac{\partial^{2} V^{h}}{\partial b_{x}^{j} \partial b_{x}^{m}}\right)+\frac{1}{2}\left(\frac{\partial V^{i}}{\partial b_{x}^{j}} \frac{\partial c_{m}^{j h}}{\partial b_{x}^{l}}+\frac{\partial V^{i}}{\partial b_{x}^{j}} \frac{\partial c_{l}^{j h}}{\partial b_{x}^{m}}\right)=0 .
\end{aligned}
$$

The conditions (23a), (23b), (23c) can be simplified by using objects $g_{i j}, c_{i j k}$ with lower indices, leading to

$$
\begin{aligned}
& g_{i j} \frac{\partial V^{j}}{\partial b_{x}^{h}}-\frac{\partial V^{j}}{\partial b_{x}^{i}} g_{j h}=0, \\
& c_{m k l} \frac{\partial V^{m}}{\partial b_{x}^{i}}+c_{m i k} \frac{\partial V^{m}}{\partial b_{x}^{l}}+c_{m l i} \frac{\partial V^{m}}{\partial b_{x}^{k}}=0, \\
& \frac{\partial^{2} V^{k}}{\partial b_{x}^{i} \partial b_{x}^{j}}=g^{k s} c_{s m j} \frac{\partial V^{m}}{\partial b_{x}^{i}}+g^{k s} c_{s m i} \frac{\partial V^{m}}{\partial b_{x}^{j}} .
\end{aligned}
$$


Indeed, lowering indices in (23a) leads to (24a). Similarly, lowering indices in (23b) and using (24a) leads to (24c). Using (23b) to eliminate secondorder derivatives in $(23 \mathrm{c})$ we get

$$
-\frac{\partial g^{i h}}{\partial b_{x}^{k}} \frac{\partial V^{k}}{\partial b_{x}^{l}}+2\left(c_{k}^{i h} \frac{\partial V^{k}}{\partial b_{x}^{l}}-\frac{\partial V^{i}}{\partial b_{x}^{k}} c_{l}^{k h}\right)-c_{l}^{i j} \frac{\partial V^{h}}{\partial b_{x}^{j}}+\frac{\partial V^{i}}{\partial b_{x}^{j}} \frac{\partial g^{j h}}{\partial b_{x}^{l}}+\frac{\partial V^{i}}{\partial b_{x}^{j}} c_{l}^{j h}=0 .
$$

Lowering indices and using (24a) again we obtain

$$
\left(g_{p s, k}+2 c_{s p k}\right) \frac{\partial V^{k}}{\partial b_{x}^{l}}+\left(-g_{s k, l}-c_{s k l}\right) \frac{\partial V^{k}}{\partial b_{x}^{p}}-c_{k p l} \frac{\partial V^{k}}{\partial b_{x}^{s}}=0 .
$$

Using (4) we obtain (24b). It remains to show that equation (23d) is a differential consequence of equations (24). In order to prove this statement we will need equation (4d). Let us differentiate (24c) with respect to $b_{x}^{l}$ and lower the index $k$ by the metric $g$. Using (4), (5), (6) we obtain

$$
\begin{aligned}
& g_{k m} \frac{\partial^{3} V^{m}}{\partial b_{x}^{j} \partial b_{x}^{i} \partial b_{x}^{l}}=g^{p q}\left(c_{k p i} c_{q m l}+c_{k p l} c_{q m i}\right) \frac{\partial V^{m}}{\partial b_{x}^{j}} \\
& \quad+g^{p q}\left(c_{k p j} c_{q m l}+c_{k p l} c_{q m j}\right) \frac{\partial V^{m}}{\partial b_{x}^{i}}+g^{p q}\left(c_{k p i} c_{q m j}+c_{k p j} c_{q m i}\right) \frac{\partial V^{m}}{\partial b_{x}^{l}} .
\end{aligned}
$$

One can show that equation (23d) can be brought to this form. Let us first bring (23d) to the form

$$
\begin{aligned}
\frac{\partial^{3} V^{k}}{\partial b_{x}^{i} \partial b_{x}^{j} \partial b_{x}^{l}}= & -\frac{1}{2} g_{j m}\left(2 c_{s}^{m k} g^{s q} c_{q p i}+\frac{\partial c_{i}^{m k}}{\partial b_{x}^{p}}+c_{i}^{m s} g^{k q} c_{q p s}\right) \frac{\partial V^{p}}{\partial b_{x}^{l}} \\
& -\frac{1}{2} g_{j m}\left(2 c_{s}^{m k} g^{s q} c_{q p l}+\frac{\partial c_{l}^{m k}}{\partial b_{x}^{p}}+c_{l}^{m s} g^{k q} c_{q p s}\right) \frac{\partial V^{p}}{\partial b_{x}^{i}} \\
& -\frac{1}{2} g^{k q} g_{j m}\left(c_{i}^{m s} c_{q p l}+c_{l}^{m s} c_{q p i}\right) \frac{\partial V^{p}}{\partial b_{x}^{s}} \\
& +\frac{1}{2} g_{s m}\left(\frac{\partial c_{i}^{s k}}{\partial b_{x}^{l}}+\frac{\partial c_{l}^{s k}}{\partial b_{x}^{i}}\right) \frac{\partial V^{m}}{\partial b_{x}^{j}} .
\end{aligned}
$$

Observe that the term $(25 \mathrm{c})$ can be rearranged as

$$
\begin{aligned}
g_{j m}\left(c_{i}^{m s} c_{q p l}\right. & \left.+c_{l}^{m s} c_{q p i}\right) \frac{\partial V^{p}}{\partial a^{s}} \\
& =g^{s b}\left(c_{b j i} c_{q p l}+c_{b j l} c_{q p i}\right) \frac{\partial V^{p}}{\partial a^{s}} \\
& =g^{s p}\left(c_{b j i} c_{q p l}+c_{b j l} c_{q p i}\right) \frac{\partial V^{b}}{\partial a^{s}} \\
& =-c_{q p l}\left(c_{b s j} \frac{\partial V^{b}}{\partial a^{i}}+c_{b i s} \frac{\partial V^{b}}{\partial a^{j}}\right)-c_{q p i}\left(c_{b s j} \frac{\partial V^{b}}{\partial a^{l}}+c_{b l s} \frac{\partial V^{b}}{\partial a^{j}}\right),
\end{aligned}
$$


where we used (24a) and (24b). Lowering indices in (25), using

$$
g_{h k} g_{j m} \frac{\partial c_{l}^{m k}}{\partial b_{x}^{p}}=\frac{\partial c_{h j l}}{\partial b_{x}^{p}}-\frac{\partial g_{h k}}{\partial b_{x}^{p}} g_{j m} c_{l}^{m k}-g_{h k} \frac{\partial g_{j m}}{\partial b_{x}^{p}} c_{l}^{m k},
$$

as well as (4), we arrive at (23d). Replacing in (24) $b_{x}^{i}$ by $u^{i}$ we obtain (11). To finish the proof, it remains to note that conditions (11) imply the existence of a (nonlocal) Hamiltonian, see Theorem 7 of Section 2.5 for explicit formulae.

\subsection{Involutivity of system (11): proof of Theorem 2}

In this section we establish the involutivity of system (11), and estimate the number of parameters in the general solution.

Theorem 2. System (11) is in involution. Its general solution depends on $\leq \frac{n(n+3)}{2}$ arbitrary constants.

Proof. We need to show that differentiation of first-order conditions (11a) and (11b) does not lead to new first-order relations. Then, we need to demonstrate consistency of second-order equations (11c). All this is a straightforward tensor algebra. Differentiating

$$
g_{i p} V_{j}^{p}=g_{j p} V_{i}^{p}
$$

we obtain

$$
g_{i p} V_{j k}^{p}+g_{i p, k} V_{j}^{p}=g_{j p} V_{i k}^{p}+g_{j p, k} V_{i}^{p} .
$$

Using (11c) we get

$$
g_{i p} g^{p s}\left[c_{s m j} V_{k}^{m}+c_{s m k} V_{j}^{m}\right]+g_{i p, k} V_{j}^{p}=g_{j p} g^{p s}\left[c_{s m k} V_{i}^{m}+c_{s m i} V_{k}^{m}\right]+g_{j p, k} V_{i}^{p} .
$$

Thus,

$$
c_{i m j} V_{k}^{m}+c_{i m k} V_{j}^{m}+g_{i p, k} V_{j}^{p}=c_{j m k} V_{i}^{m}+c_{j m i} V_{k}^{m}+g_{j p, k} V_{i}^{p},
$$

or, relabelling indices,

$$
c_{i p j} V_{k}^{p}+c_{i p k} V_{j}^{p}+g_{i p, k} V_{j}^{p}=c_{j p k} V_{i}^{p}+c_{j p i} V_{k}^{p}+g_{j p, k} V_{i}^{p} .
$$

This can be rewritten in the form

$$
\left(c_{i p j}-c_{j p i}\right) V_{k}^{p}+\left(c_{i p k}+g_{i p, k}\right) V_{j}^{p}-\left(c_{j p k}+g_{j p, k}\right) V_{i}^{p}=0 .
$$


Taking into account (4a) we obtain

$$
\left(c_{i p j}-c_{j p i}\right) V_{k}^{p}-c_{p i k} V_{j}^{p}+c_{p j k} V_{i}^{p}=0 .
$$

Due to (4b) we can rewrite this as

$$
\left(c_{i p j}-c_{j p i}\right) V_{k}^{p}+c_{p k i} V_{j}^{p}+c_{p j k} V_{i}^{p}=0 .
$$

Using (11b) we obtain

$$
\left(c_{i p j}-c_{j p i}\right) V_{k}^{p}=c_{p i j} V_{k}^{p} .
$$

It remains to note that the equality $c_{i p j}-c_{j p i}=c_{p i j}$ holds identically due to the cyclic condition (4c). Thus, differentiation of (11a) does not lead to new first-order relations.

Similarly, differentiating (11b) we obtain

$$
c_{m k l, j} V_{i}^{m}+c_{m k l} V_{i j}^{m}+c_{m i k, j} V_{l}^{m}+c_{m i k} V_{l j}^{m}+c_{m l i, j} V_{k}^{m}+c_{m l i} V_{k j}^{m}=0 .
$$

The substitution of (4d) and (11c) gives

$$
\begin{aligned}
& -g^{p q} c_{p m j} c_{q k l} V_{i}^{m}+c_{m k l} g^{m s}\left(c_{s p i} V_{j}^{p}+c_{s p j} V_{i}^{p}\right) \\
& -g^{p q} c_{p m j} c_{q i k} V_{l}^{m}+c_{m i k} g^{m s}\left(c_{s p l} V_{j}^{p}+c_{s p j} V_{l}^{p}\right) \\
& -g^{p q} c_{p m j} c_{q l i} V_{k}^{m}+c_{m l i} g^{m s}\left(c_{s p k} V_{j}^{p}+c_{s p j} V_{k}^{p}\right)=0 .
\end{aligned}
$$

Note that all terms apart from those containing $V_{j}^{p}$ cancel, leading to

$$
g^{m s}\left(c_{m k l} c_{s p i}+c_{m i k} c_{s p l}+c_{m l i} c_{s p k}\right) V_{j}^{p}=0 .
$$

Due to (4d), (4b) this expression can be rewritten as

$$
-\left(c_{k p i, l}+c_{p i k, l}+c_{i k p, l}\right) V_{j}^{p}=0,
$$

which is an identity due to (4c).

The compatibility of second-order relations (11c) can be shown as follows. Computation of the consistency condition $V_{i j, k}^{p}=V_{k j, i}^{p}$ gives

$$
\begin{aligned}
& g_{, k}^{p s}\left[c_{s m j} V_{i}^{m}+c_{s m i} V_{j}^{m}\right] \\
&+g^{p s}\left[c_{s m j} V_{i k}^{m}+c_{s m j, k} V_{i}^{m}+c_{s m i} V_{j k}^{m}+c_{s m i, k} V_{j}^{m}\right] \\
& \quad=g_{, i}^{p s}\left[c_{s m j} V_{k}^{m}+c_{s m k} V_{j}^{m}\right] \\
& \quad+g^{p s}\left[c_{s m j} V_{i k}^{m}+c_{s m j, i} V_{k}^{m}+c_{s m k} V_{i j}^{m}+c_{s m k, i} V_{j}^{m}\right] .
\end{aligned}
$$


Cancelling terms with $V_{i k}^{m}$ results in a simplified expression,

$$
\begin{aligned}
& g_{, k}^{p s}\left[c_{s m j} V_{i}^{m}+c_{s m i} V_{j}^{m}\right]+g^{p s}\left[c_{s m j, k} V_{i}^{m}+c_{s m i} V_{j k}^{m}+c_{s m i, k} V_{j}^{m}\right] \\
= & g_{, i}^{p s}\left[c_{s m j} V_{k}^{m}+c_{s m k} V_{j}^{m}\right]+g^{p s}\left[c_{s m j, i} V_{k}^{m}+c_{s m k} V_{i j}^{m}+c_{s m k, i} V_{j}^{m}\right] .
\end{aligned}
$$

Contraction with $g_{p q}$ gives

$$
\begin{aligned}
& g_{p q} g_{, k}^{p s}\left[c_{s m j} V_{i}^{m}+c_{s m i} V_{j}^{m}\right]+c_{q m j, k} V_{i}^{m}+c_{q m i} V_{j k}^{m}+c_{q m i, k} V_{j}^{m} \\
= & g_{p q} g_{, i}^{p s}\left[c_{s m j} V_{k}^{m}+c_{s m k} V_{j}^{m}\right]+c_{q m j, i} V_{k}^{m}+c_{q m k} V_{i j}^{m}+c_{q m k, i} V_{j}^{m} .
\end{aligned}
$$

Taking into account (11c) along with the identity $g_{p q} g_{, k}^{p s}=-g^{s p} g_{p q, k}$ we get

$$
\begin{aligned}
& -g^{s p} g_{p q, k}\left[c_{s m j} V_{i}^{m}+c_{s m i} V_{j}^{m}\right]+c_{q m j, k} V_{i}^{m} \\
& \quad+c_{q p i} g^{p s}\left[c_{s m j} V_{k}^{m}+c_{s m k} V_{j}^{m}\right]+c_{q m i, k} V_{j}^{m} \\
& =-g^{s p} g_{p q, i}\left[c_{s m j} V_{k}^{m}+c_{s m k} V_{j}^{m}\right]+c_{q m j, i} V_{k}^{m} \\
& \quad+c_{q p k} g^{p s}\left[c_{s m j} V_{i}^{m}+c_{s m i} V_{j}^{m}\right]+c_{q m k, i} V_{j}^{m} .
\end{aligned}
$$

Rearrangement gives

$$
\begin{aligned}
& {\left[c_{q m j, k}-c_{q p k} g^{p s} c_{s m j}-g^{s p} g_{p q, k} c_{s m j}\right] V_{i}^{m}} \\
& \quad+\left[c_{q p i} g^{p s} c_{s m j}+g^{s p} g_{p q, i} c_{s m j}-c_{q m j, i}\right] V_{k}^{m} \\
& +\left[c_{q m i, k}-g^{s p} g_{p q, k} c_{s m i}+c_{q p i} g^{p s} c_{s m k}+g^{s p} g_{p q, i} c_{s m k}\right. \\
& \left.\quad-c_{q p k} g^{p s} c_{s m i}-c_{q m k, i}\right] V_{j}^{m}=0 .
\end{aligned}
$$

Taking into account (4a) we obtain

$$
\begin{gathered}
\left(c_{q m j, k}+g^{s p} c_{s m j} c_{p q k}\right) V_{i}^{m}-\left(c_{q m j, i}+g^{s p} c_{s m j} c_{p q i}\right) V_{k}^{m} \\
+\left[\left(c_{q m i, k}+g^{s p} c_{s m i} c_{p q k}\right)-\left(c_{q m k, i}+g^{s p} c_{s m k} c_{p q i}\right)\right] V_{j}^{m}=0,
\end{gathered}
$$

which is an identity due to $(4 \mathrm{~d})$.

Thus, system (11) is in involution. Since equations (11c) express all second-order partial derivatives of $V^{i}$, the general solution depends on no more than $n+n^{2}$ parameters (values of $V^{i}$ and first-order derivatives thereof). However, relations (11a) impose $\frac{n(n-1)}{2}$ independent constraints on firstorder derivatives of $V^{i}$. Thus, the general solution depends on no more than $n+n^{2}-\frac{n(n-1)}{2}=\frac{n(n+3)}{2}$ arbitrary constants: the inequality is due to the extra first-order relations (11b) that are not so easy to control. Examples show that solution spaces to equations (11) for different third-order Hamiltonian operators (with the same number of components) may have different dimensions. In particular, the maximal possible dimension, $\frac{n(n+3)}{2}$, corresponds to constant-coefficient operators $\left(g_{i j}=\right.$ const, $\left.c_{i j k}=0\right)$. 


\subsection{Integration of system (11): proof of Theorem 3}

It is quite remarkable that system (11) for the fluxes $V^{i}$, which is a linear involutive system with non-constant coefficients, can be integrated in closed form. Let us recall that the metric $g$ defining Hamiltonian operator (3) can be represented in factorised form (7), $g_{i j}=\phi_{\beta \gamma} \psi_{i}^{\beta} \psi_{j}^{\gamma}$, where $\phi_{\beta \gamma}$ is a nondegenerate constant symmetric matrix, and $\psi_{k}^{\gamma}=\psi_{k m}^{\gamma} u^{m}+\omega_{k}^{\gamma}$; here $\psi_{k m}^{\gamma}$ and $\omega_{k}^{\gamma}$ are constants such that $\psi_{k m}^{\gamma}=-\psi_{m k}^{\gamma}$. These constants satisfy a set of quadratic relations (9), (10). Using relations (7) - (10), one can show that in the new variables $W^{\gamma}$ defined as

$$
W^{\gamma}=\psi_{k}^{\gamma} V^{k}
$$

system (11) takes the form

$$
\begin{aligned}
& \phi_{\beta \gamma}\left[\psi_{i k}^{\beta} W^{\gamma}+\psi_{k}^{\beta} W_{i}^{\gamma}-\psi_{i}^{\beta} W_{k}^{\gamma}\right]=0, \\
& \phi_{\beta \gamma}\left[\psi_{i j}^{\beta} W_{k}^{\gamma}+\psi_{j k}^{\beta} W_{i}^{\gamma}+\psi_{k i}^{\beta} W_{j}^{\gamma}\right]=0, \\
& W_{i j}^{\gamma}=0,
\end{aligned}
$$

where lower indices of $W^{\gamma}$ denote partial derivatives. The last condition implies that $W^{\gamma}$ are linear functions,

$$
W^{\gamma}=\eta_{m}^{\gamma} u^{m}+\xi^{\gamma}
$$

while the first two conditions imply that the constants $\eta_{m}^{\gamma}$ and $\xi^{\gamma}$ satisfy a linear system

$$
\begin{aligned}
\phi_{\beta \gamma}\left[\psi_{i j}^{\beta} \eta_{k}^{\gamma}+\psi_{j k}^{\beta} \eta_{i}^{\gamma}+\psi_{k i}^{\beta} \eta_{j}^{\gamma}\right] & =0, \\
\phi_{\beta \gamma}\left[\psi_{i k}^{\beta} \xi^{\gamma}+\omega_{k}^{\beta} \eta_{i}^{\gamma}-\omega_{i}^{\beta} \eta_{k}^{\gamma}\right] & =0 .
\end{aligned}
$$

Thus, finding conservative Hamiltonian systems for a given third-order Hamiltonian operator (3) is reduced to linear algebra. Conversely, given conservative system (1), the reconstruction of the associated Hamiltonian representation from system (11) reduces to a linear system for the coefficients of a Monge metric. The above representation implies the following result.

Theorem 3. For Hamiltonian system (1) the following conditions hold:

- The corresponding congruence (2) is linear.

- System (1) is linearly degenerate and belongs to the Temple class. 
- The fluxes $V^{i}$ are rational functions of the form

$$
V^{i}=\frac{Q^{i}}{\operatorname{det} \psi},
$$

where det $\psi$ is a polynomial of degree $n-1$ defining the singular surface, and $Q^{i}$ are polynomials of degree $n$.

Proof. Linearity of the congruence can be demonstrated as follows. Substituting $W^{\gamma}=\eta_{m}^{\gamma} u^{m}+\xi^{\gamma}$ and $\psi_{k}^{\gamma}=\psi_{k m}^{\gamma} u^{m}+\omega_{k}^{\gamma}$ into the formula $W^{\gamma}=$ $\psi_{k}^{\gamma} V^{k}$ we obtain a linear relation in the Plücker coordinates (note the skewsymmetry condition $\psi_{k m}^{\gamma}=-\psi_{m k}^{\gamma}$ ),

$$
\frac{1}{2} \psi_{k m}^{\gamma}\left(u^{m} V^{k}-u^{k} V^{m}\right)+\omega_{k}^{\gamma} V^{k}-\eta_{m}^{\gamma} u^{m}-\xi^{\gamma}=0 .
$$

This proves the linearity. Linear degeneracy and the Temple property of system (1) follows from the linearity of the corresponding congruence [2]. Finally, solving the equations $W^{\gamma}=\psi_{k}^{\gamma} V^{k}$ for $V^{k}$ implies $V^{k}=\psi_{\gamma}^{k} W^{\gamma}$ where $\psi_{\gamma}^{k}$ is the inverse matrix to $\psi_{k}^{\gamma}$. Thus, $V^{k}$ will have $\operatorname{det} \psi$ in the denominator, while numerators will be polynomials of degree $n$.

\subsection{Projective invariance: proof of Theorem 4}

In this section we show that third-order Hamiltonian formalism (3) is invariant under reciprocal transformations (15). This is in contrast with the case of first-order Hamiltonian structures of Dubrovin-Novikov type, which generally become nonlocal after a reciprocal transformation $[12,14]$.

Theorem 4. The class of conservative systems (1) possessing third-order Hamiltonian formulation (3) is invariant under reciprocal transformations (15).

Proof. A general reciprocal transformation (15) can be represented as a composition,

$$
(x \text {-transformation }) \circ(x \leftrightarrow t) \circ(x \text {-transformation }),
$$

where $x$-transformation is a reciprocal transformation changing the variable $x$ only, and $x \leftrightarrow t$ denotes the 'inversion', that is, the interchange of $x$ and $t$. The invariance of Hamiltonian formalism (3) under $x$-transformations was established in [15]. Thus, it remains to show that third-order Hamiltonian formalism (3) is invariant under the inversion. Under this transformation, 
the new dependent variables and the new fluxes are defined as $\tilde{u}^{i}=V^{i}, \tilde{V}^{i}=$ $u^{i}$, respectively. Recall that system (1) possesses Hamiltonian operator (3) if the following conditions are satisfied:

1. Metric $g$ of Hamiltonian operator (3) possesses factorised form (7), $g_{i j}=\phi_{\beta \gamma} \psi_{i}^{\beta} \psi_{j}^{\gamma}$, where $\phi_{\beta \gamma}$ is a constant symmetric matrix and $\psi_{k}^{\gamma}=$ $\psi_{k m}^{\gamma} u^{m}+\omega_{k}^{\gamma}$; here the constants $\omega_{k}^{\gamma}$ and skew-symmetric $\psi_{k m}^{\gamma}$ satisfy relations $(9),(10)$.

2. The functions $\psi_{k}^{\gamma} V^{k}$ are linear in $u: \psi_{k}^{\gamma} V^{k}=\eta_{m}^{\gamma} u^{m}+\xi^{\gamma}$, where the constants $\eta_{m}^{\gamma}, \xi^{\gamma}$ satisfy relations (30).

We claim that the 'inverted' system is also Hamiltonian, and the metric of the transformed Hamiltonian operator is given by

$$
\tilde{g}_{i j}=V_{i}^{m} g_{m n} V_{j}^{n}
$$

note that this transformation rule is identical to that for first-order Hamiltonian operators of Dubrovin-Novikov type. Thus, we have to demonstrate the following:

1. Metric $\tilde{g}$ of the transformed Hamiltonian operator possesses factorised form $\tilde{g}_{i j}=\tilde{\phi}_{\beta \gamma} \tilde{\psi}_{i}^{\beta} \tilde{\psi}_{j}^{\gamma}$, where $\tilde{\phi}_{\beta \gamma}$ is a constant symmetric matrix, and $\tilde{\psi}_{k}^{\gamma}=\tilde{\psi}_{k m}^{\gamma} V^{m}+\tilde{\omega}_{k}^{\gamma}$; here $\tilde{\omega}_{k}^{\gamma}$ and skew-symmetric $\tilde{\psi}_{k m}^{\gamma}$ must satisfy relations (9), (10).

2. The expressions $\tilde{\psi}_{k}^{\gamma} u^{k}$ are linear in $V: \tilde{\psi}_{k}^{\gamma} u^{k}=\tilde{\eta}_{m}^{\gamma} V^{m}+\tilde{\xi}^{\gamma}$, where the constants $\tilde{\eta}_{m}^{\gamma}, \tilde{\xi}^{\gamma}$ satisfy relations (30).

We claim that this is indeed the case, furthermore,

$$
\tilde{\phi}_{\beta \gamma}=\phi_{\beta \gamma}, \quad \tilde{\psi}_{k m}^{\gamma}=\psi_{k m}^{\gamma}, \quad \tilde{\omega}_{k}^{\gamma}=\eta_{k}^{\gamma}, \quad \tilde{\eta}_{k}^{\gamma}=\omega_{k}^{\gamma}, \quad \tilde{\xi}^{\gamma}=-\xi^{\gamma},
$$

note that the constants with tilde's satisfy the same relations (9), (10), (30).

To establish part 1 we proceed as follows. Differentiating the relation $W^{\gamma}=\psi_{k}^{\gamma} V^{k}$ with respect to $u^{m}$ we obtain $\eta_{m}^{\gamma}=\psi_{k}^{\gamma} V_{m}^{k}+\psi_{k m}^{\gamma} V^{k}$. Solving for $V_{m}^{k}$ gives $V_{m}^{k}=\psi_{\gamma}^{k} \eta_{m}^{\gamma}-\psi_{\gamma}^{k} \psi_{s m}^{\gamma} V^{s}$, where $\psi_{\gamma}^{k}$ is the inverse matrix to $\psi_{k}^{\gamma}$. Thus, using (32),

$$
\begin{gathered}
\tilde{g}_{i j}=V_{i}^{m} g_{m k} V_{j}^{k}=\left(\psi_{\gamma}^{m} \eta_{i}^{\gamma}-\psi_{\gamma}^{m} \psi_{r i}^{\gamma} V^{r}\right) g_{m k}\left(\psi_{\tau}^{k} \eta_{j}^{\tau}-\psi_{\tau}^{k} \psi_{s j}^{\tau} V^{s}\right) \\
=\left(\psi_{\gamma}^{m} \eta_{i}^{\gamma}-\psi_{\gamma}^{m} \psi_{r i}^{\gamma} V^{r}\right) \psi_{m}^{\beta} \phi_{\beta \gamma} \psi_{k}^{\gamma}\left(\psi_{\tau}^{k} \eta_{j}^{\tau}-\psi_{\tau}^{k} \psi_{s j}^{\tau} V^{s}\right)
\end{gathered}
$$


$=\left(\eta_{i}^{\beta}-\psi_{r i}^{\beta} V^{r}\right) \phi_{\beta \gamma}\left(\eta_{j}^{\gamma}-\psi_{s j}^{\gamma} V^{s}\right)=\left(\eta_{i}^{\beta}+\psi_{i r}^{\beta} V^{r}\right) \phi_{\beta \gamma}\left(\eta_{j}^{\gamma}+\psi_{j s}^{\gamma} V^{s}\right)=\tilde{\psi}_{i}^{\beta} \phi_{\beta \gamma} \tilde{\psi}_{j}^{\gamma}$, which is the required formula. Finally, for part 2, it is a simple exercise to verify that the relation $\tilde{\psi}_{k}^{\gamma} u^{k}=\tilde{\eta}_{m}^{\gamma} V^{m}+\tilde{\xi}^{\gamma}$ follows from $\psi_{k}^{\gamma} V^{k}=\eta_{m}^{\gamma} u^{m}+$ $\xi^{\gamma}$.

\subsection{Casimirs, Momentum, Hamiltonian}

Given system (1) satisfying conditions (11), in this section we derive explicit formulae for the corresponding Casimirs, Momentum and the Hamiltonian. To do so we introduce the substitution $u^{i}=b_{x}^{i}$ transforming system (1) into (non-quasilinear) first-order form (21),

$$
b_{t}^{i}=V^{i}\left(\mathbf{b}_{x}\right) .
$$

In variables $b^{i}$, operator (3) takes first-order form (22). Using $g_{i j}=\phi_{\beta \gamma} \psi_{i}^{\beta} \psi_{j}^{\gamma}$ we can rewrite it in factorised form,

$$
P^{i j}=-\phi^{\beta \gamma} \psi_{\beta}^{i} \partial_{x} \psi_{\gamma}^{j},
$$

recall that $\psi_{\beta}^{i}$ is the inverse matrix to $\psi_{i}^{\beta}$.

Theorem 7. System (21) can be represented in Hamiltonian form,

$$
b_{t}^{i}=V^{i}\left(\mathbf{b}_{x}\right)=P^{i j} \frac{\delta H}{\delta b^{j}},
$$

with the local Hamiltonian

$$
\begin{aligned}
H=\int h d x & =-\int \phi_{\beta \gamma}\left[\left(\frac{1}{3} \eta_{p}^{\gamma} \psi_{q m}^{\beta} b_{x}^{m}+\frac{1}{2} \omega_{p}^{\beta} \eta_{q}^{\gamma}\right) b^{p} b^{q}\right. \\
& \left.+x \xi^{\gamma}\left(\frac{1}{2} \psi_{p q}^{\beta} b^{p} b_{x}^{q}+\omega_{q}^{\beta} b^{q}\right)\right] d x,
\end{aligned}
$$

note the explicit $x$-dependence. The $n$ Casimirs are given by

$$
C^{\alpha}=\int c^{\alpha} d x=\int\left(\frac{1}{2} \psi_{m k}^{\alpha} b_{x}^{k}+\omega_{m}^{\alpha}\right) b^{m} d x .
$$

The Momentum has the form

$$
M=\int m d x=-\int\left(\frac{1}{3} \phi_{\beta \gamma} \omega_{q}^{\beta} \psi_{p m}^{\gamma} b_{x}^{m}+\frac{1}{2} \phi_{\beta \gamma} \omega_{p}^{\beta} \omega_{q}^{\gamma}\right) b^{p} b^{q} d x
$$


Remark. In the particular case $\xi=0$, equations (29), (30), (33) were obtained in [29]. If $\xi \neq 0$, the corresponding Hamiltonian density $h$ has explicit $x$-dependence. It may be more than just a curiosity that all known integrable systems (1) with Hamiltonian structure (3) admit a local compatible first-order Hamiltonian operator iff $h$ has no explicit $x$-dependence.

Proof. Using relations (30), one obtains the following expression for the variational derivative of $H$,

$$
\frac{\delta H}{\delta b^{j}}=-\phi_{\beta \gamma}\left(\psi_{j p}^{\beta} b_{x}^{p}+\omega_{j}^{\beta}\right)\left(\eta_{q}^{\gamma} b^{q}+\xi^{\gamma} x\right)=-\phi_{\beta \gamma} \psi_{j}^{\beta}\left(\eta_{q}^{\gamma} b^{q}+\xi^{\gamma} x\right) .
$$

Thus,

$$
\begin{gathered}
b_{t}^{i}=P^{i j} \frac{\delta H}{\delta b^{j}}=-\phi^{\beta \gamma} \psi_{\beta}^{i} \partial_{x} \psi_{\gamma}^{j} \frac{\delta H}{\delta b^{j}}=\phi^{\beta \gamma} \psi_{\beta}^{i} \partial_{x} \psi_{\gamma}^{j} \phi_{\mu \nu} \psi_{j}^{\mu}\left(\eta_{q}^{\nu} b^{q}+\xi^{\nu} x\right) \\
=\phi^{\beta \gamma} \psi_{\beta}^{i} \partial_{x} \phi_{\gamma \nu}\left(\eta_{q}^{\nu} b^{q}+\xi^{\nu} x\right)=\phi^{\beta \gamma} \psi_{\beta}^{i} \phi_{\gamma \nu}\left(\eta_{q}^{\nu} b_{x}^{q}+\xi^{\nu}\right) \\
=\psi_{\nu}^{i}\left(\eta_{q}^{\nu} b_{x}^{q}+\xi^{\nu}\right)=\psi_{\nu}^{i} W^{\nu}=V^{i}\left(\mathbf{b}_{x}\right),
\end{gathered}
$$

as required. Similarly, variational derivatives of the Casimirs are

$$
\frac{\delta C^{\alpha}}{\delta b^{j}}=\psi_{j k}^{\alpha} b_{x}^{k}+\omega_{j}^{\alpha}=\psi_{j}^{\alpha},
$$

so that

$$
P^{i j} \frac{\delta C^{\alpha}}{\delta b^{j}}=-\phi^{\beta \gamma} \psi_{\beta}^{i} \partial_{x} \psi_{\gamma}^{j} \frac{\delta C^{\alpha}}{\delta b^{j}}=-\phi^{\beta \gamma} \psi_{\beta}^{i} \partial_{x} \psi_{\gamma}^{j} \psi_{j}^{\alpha}=-\phi^{\beta \gamma} \psi_{\beta}^{i} \partial_{x} \delta_{\gamma}^{\alpha}=0 .
$$

Finally, using (10), one computes variational derivatives of the Momentum,

$$
\frac{\delta M}{\delta b^{j}}=-\phi_{\beta \gamma} \psi_{j}^{\beta} \omega_{m}^{\gamma} b^{m}=-\phi_{\beta \gamma} \psi_{j}^{\beta} \partial_{x}^{-1} \psi_{m}^{\gamma} b_{x}^{m},
$$

thus,

$$
P^{i j} \frac{\delta M}{\delta b^{j}}=-\phi^{\beta \gamma} \psi_{\beta}^{i} \partial_{x} \psi_{\gamma}^{j} \frac{\delta M}{\delta b^{j}}=b_{x}^{i},
$$

as required. Note that in the original variables $u^{i}$, all of the above densities become nonlocal. 


\subsection{Algebraic reformulation of conditions (9), (10), (30)}

In this section we demonstrate that algebraic constraints (9), (10), (30) can be represented in a compact invariant form which substantially simplifies their analysis. Let us note that lines (2) pass through the points $y^{i}=$ $u^{i}, y^{n+1}=1, y^{n+2}=0$ and $y^{i}=V^{i}, y^{n+1}=0, y^{n+2}=1$, respectively. The corresponding Plücker coordinates, which are $2 \times 2$ minors of the $2 \times(n+2)$ matrix

$$
\left(\begin{array}{ccccc}
u^{i} & \ldots & u^{n} & 1 & 0 \\
V^{i} & \ldots & V^{n} & 0 & 1
\end{array}\right)
$$

can be arranged into $(n+2) \times(n+2)$ skew-symmetric matrix,

$$
Y=\left(\begin{array}{ccc|cc} 
& & & -V^{1} & u^{1} \\
& U & & \vdots \\
& & & -V^{n} & u^{n} \\
\hline V^{1} & \ldots & V^{n} & 0 & 1 \\
-u^{1} & \ldots & -u^{n} & -1 & 0
\end{array}\right),
$$

here $U$ is the skew-symmetric matrix with entries $u^{i} V^{j}-u^{j} V^{i}$. In this notation, relations (31) can be represented as

$$
\operatorname{tr} Y A^{\gamma}=0,
$$

where $(n+2) \times(n+2)$ skew-symmetric matrices $A^{\gamma}$ are defined as

$$
A^{\gamma}=\left(\begin{array}{ccc|cc} 
& & & \omega_{1}^{\gamma} & \eta_{1}^{\gamma} \\
& \frac{1}{2} \psi^{\gamma} & & \vdots & \vdots \\
& & \omega_{n}^{\gamma} & \eta_{n}^{\gamma} \\
\hline-\omega_{1}^{\gamma} & \ldots & -\omega_{n}^{\gamma} & 0 & \xi^{\gamma} \\
-\eta_{1}^{\gamma} & \ldots & -\eta_{n}^{\gamma} & -\xi^{\gamma} & 0
\end{array}\right),
$$

here $\psi^{\gamma}$ is the skew-symmetric matrix with entries $\psi_{i j}^{\gamma}$. What is remarkable, relations (9), (10), (30) compactify into a single relation

$$
\phi_{\beta \gamma} A^{\beta} \wedge A^{\gamma}=0,
$$

where each $A^{\gamma}$ is interpreted as a 2 -form.

\subsection{Classification results: proof of Theorems 5, 6}

In this Section we summarise the classification of 2- and 3-component Hamiltonian systems of conservation laws based on the classification of linear congruences in $\mathbb{P}^{3}$ and $\mathbb{P}^{4}$. 
Theorem 5. For $n=2$, every Hamiltonian system of conservation laws is linearisable (that is, equivalent to 2-component case of Example 1 from Section 1.4).

Proof. Every linear congruence in $\mathbb{P}^{3}$ consists of bisecants of two skew lines. Modulo projective transformations, any such congruence can be brought to the form

$$
y^{1}=u^{1} y^{3}+u^{2} y^{4}, \quad y^{2}=u^{2} y^{3}+u^{1} y^{4},
$$

where $y^{i}$ are homogeneous coordinates in $\mathbb{P}^{3}$. In the affine chart $y^{4}=1$, the skew lines in question can be defined as $y^{3}=1, y^{1}=y^{2}$ and $y^{3}=-1, y^{1}=$ $-y^{2}$, respectively. The corresponding system of conservation laws is clearly linear,

$$
u_{t}^{1}=u_{x}^{2}, \quad u_{t}^{2}=u_{x}^{1},
$$

which is a particular case of Example 1.

Theorem 6. For $n=3$, every Hamiltonian system of conservation laws is either linearisable (that is, equivalent to 3-component case of Example 1 from Section 1.4), or equivalent to the system of WDVV equations (Example 2 from Section 1.4).

Proof. Linear congruences in $\mathbb{P}^{4}$ were classified by Castelnuovo in [5]. In our presentation we follow [2], and use $\left(y^{1}: \cdots: y^{5}\right)$ for homogeneous coordinates in $\mathbb{P}^{4}$. Over $\mathbb{C}$, every linear congruence in $\mathbb{P}^{4}$ can be brought to one of the four normal forms:

- Generic case: the focal variety is a generic projection of the Veronese surface $V^{2} \subset \mathbb{P}^{5}$ into $\mathbb{P}^{4}$ :

$$
y^{1}=u^{1} y^{4}+u^{2} y^{5}, \quad y^{2}=u^{2} y^{4}+u^{3} y^{5}, \quad y^{3}=u^{3} y^{4}+\left(\left(u^{2}\right)^{2}-u^{1} u^{3}\right) y^{5} .
$$

The corresponding system,

$$
u_{t}^{1}=u_{x}^{2}, \quad u_{t}^{2}=u_{x}^{3}, \quad u_{t}^{3}=\left(\left(u^{2}\right)^{2}-u^{1} u^{3}\right)_{x},
$$

does not possess Riemann invariants (Example 2).

- The focal variety is reducible, and consists of a cubic scroll and a plane which intersects the cubic scroll along its directrix:

$$
y^{1}=u^{1} y^{4}+u^{2} y^{5}, \quad y^{2}=u^{2} y^{4}+u^{3} y^{5}, \quad y^{3}=u^{3} y^{4}+\frac{u^{2} u^{3}}{u^{1}} y^{5} .
$$


The corresponding system,

$$
u_{t}^{1}=u_{x}^{2}, \quad u_{t}^{2}=u_{x}^{3}, \quad u_{t}^{3}=\left(\frac{u^{2} u^{3}}{u^{1}}\right)_{x},
$$

possesses one Riemann invariant. One can show that this system does not possess non-degenerate third-order Hamiltonian structures.

- The focal variety is reducible, and consists of a two-dimensional quadric and two planes which intersect the quadric along rectilinear generators of different families:

$$
y^{1}=u^{1} y^{4}+u^{2} y^{5}, \quad y^{2}=u^{2} y^{4}+u^{3} y^{5}, \quad y^{3}=u^{3} y^{4}+\frac{\left(u^{3}\right)^{2}-1}{u^{2}} y^{5} .
$$

The corresponding system,

$$
u_{t}^{1}=u_{x}^{2}, \quad u_{t}^{2}=u_{x}^{3}, \quad u_{t}^{3}=\left(\frac{\left(u^{3}\right)^{2}-1}{u^{2}}\right)_{x},
$$

possesses two Riemann invariants. One can show that this system does not possess non-degenerate third-order Hamiltonian structures.

- The focal variety consists of 3 planes in general position:

$$
y^{1}=u^{1} y^{4}+u^{2} y^{5}, \quad y^{2}=u^{2} y^{4}+u^{3} y^{5}, \quad y^{3}=u^{3} y^{4}+u^{2} y^{5} .
$$

The corresponding system is linear:

$$
u_{t}^{1}=u_{x}^{2}, \quad u_{t}^{2}=u_{x}^{3}, \quad u_{t}^{3}=u_{x}^{2},
$$

(Example 1).

Note that the number of planar components of the focal variety equals the number of Riemann invariants of the associated system [2].

\section{Concluding remarks}

The classification of $n$-component Hamiltonian systems of conservation laws has been reduced to the following algebraic problem: for a vector space $W$ of dimension $n+2$, classify $n$-dimensional subspaces $A \subset \Lambda^{2}(W)$ satisfying a relation

$$
\phi_{\beta \gamma} A^{\beta} \wedge A^{\gamma}=0
$$

where $A^{\alpha}$ is a basis of $A$ and $\phi$ is symmetric and non-degenerate. This gives rise to the following natural questions: 
- Classify normal forms of such subspaces $A$, at least for $n=4$. This would provide explicit coordinate representation of Hamiltonian systems of conservation laws.

- Classify subspaces $A$ corresponding to integrable systems of conservation laws (note that for $n=2,3$ all Hamiltonian systems are automatically integrable). We emphasise that for $n \geq 4$ the integrability is no longer the case in general. We expect that Example 3 from Section 1.4 will play a key role in this classification.

We hope to return to these questions elsewhere.

\section{Acknowledgements}

We thank R. Chirivì, N. Hitchin, A. King, J.S. Krasil'shchik, L. Manivel, and A.M. Verbovetsky for clarifying discussions. We acknowledge financial support from GNFM of the Istituto Nazionale di Alta Matematica, the Istituto Nazionale di Fisica Nucleare by IS-CSN4 Mathematical Methods of Nonlinear Physics, and the Dipartimento di Matematica e Fisica "E. De Giorgi" of the Università del Salento. MVP's work was partially supported by the grant of the Presidium of RAS 'Fundamental Problems of Nonlinear Dynamics'.

\section{References}

[1] S.I. Agafonov and E.V. Ferapontov, Systems of conservation laws from the point of view of the projective theory of congruences, Izv. RAN, ser. mat. 60 (1996) N.6, 3-30.

[2] S. I. Agafonov and E. V. Ferapontov, Systems of conservation laws of Temple class, equations of associativity and linear congruences in $P^{4}$, Manuscripta Math. 106, p. 4 (2001) 461-488.

[3] S.I. Agafonov and E.V. Ferapontov, Integrable four-component systems of conservation laws and linear congruences in $\mathbb{P}^{5}$, Glasgow Math. J. 47A (2005) 17-32.

[4] A.V. Balandin, G.V. Potemin, On non-degenerate differentialgeometric Poisson brackets of third order, Russian Mathematical Surveys 56 No. 5 (2001) 976-977. 
[5] G. Castelnuovo, Ricerche di geometria della retta nello spazio a quattro dimensioni, Ven. Ist. Atti. (7), II (1891) 855-901.

[6] P. De Poi, E. Mezzetti, Linear congruences and hyperbolic systems of conservation laws. Projective varieties with unexpected properties, 209-230, Walter de Gruyter GmbH \& Co. KG, Berlin, 2005.

[7] P. De Poi, E. Mezzetti, Congruences of lines in $\mathbb{P}^{5}$, quadratic normality, and completely exceptional Monge-Ampère equations, Geom. Dedicata 131 (2008) 213-230.

[8] P. De Poi, E. Mezzetti, On a class of first order congruences of lines, Bull. Belg. Math. Soc. Simon Stevin 16, no. 5 (2009), Linear systems and subschemes, 805-821.

[9] P.W. Doyle, Differential geometric Poisson bivectors in one space variable, J. Math. Phys. 34 No. 4 (1993) 1314-1338.

[10] B.A. Dubrovin and S.P. Novikov, Poisson brackets of hydrodynamic type, Soviet Math. Dokl. 30 No. 3 (1984), 651-2654.

[11] B.A. Dubrovin, Geometry of 2D topological field theories, Lecture Notes in Mathematics, V.1620, Berlin, Springer, 120-348.

[12] E.V. Ferapontov, Nonlocal Hamiltonian operators of hydrodynamic type: differential geometry and applications, Amer. Math. Soc. Transl. (2) 170 (1995) 33-58.

[13] E.V. Ferapontov, C.A.P. Galvao, O. Mokhov, Y. Nutku, BiHamiltonian structure of equations of associativity in 2-d topological field theory, Comm. Math. Phys. 186 (1997) 649-669.

[14] E.V. Ferapontov, M. V. Pavlov, Reciprocal transformations of Hamiltonian operators of hydrodynamic type: non-local Hamiltonian formalism for linearly degenerate systems, J. Math. Phys. 44 No. 3 (2003) 11501172 .

[15] E.V. Ferapontov, M.V. Pavlov, R.F. Vitolo, Projective-geometric aspects of homogeneous third-order Hamiltonian operators, J. Geom. Phys. 85 (2014) 16-28, DOI: 10.1016/j.geomphys.2014.05.027.

[16] E.V. Ferapontov, M.V. Pavlov and R.F. Vitolo, Towards the classification of homogeneous third-order Hamiltonian operators, IMRN (2016) doi:10.1093/imrn/rnv369; arXiv:1508.02752. 
[17] E.V. Ferapontov, Isoparametric hypersurfaces in spheres, integrable nondiagonalizable systems of hydrodynamic type, and N-wave systems, Diff. Geom. Appl. 5 (1995) 335-369.

[18] E. Getzler, A Darboux theorem for Hamiltonian operators in the formal calculus of variations, Duke J. Math. 111 (2002), 535-560.

[19] J. Kalayci, Y. Nutku, Bi-Hamiltonian structure of a WDVV equation in 2d topological field theory, Phys. Lett. A 227 (1997), 177-182.

[20] J. Kalayci, Y. Nutku, Alternative bi-Hamiltonian structures for WDVV equations of associativity, J. Phys. A: Math. Gen. 31 (1998) 723-734.

[21] A. Jeffrey, Quasilinear hyperbolic systems and waves, Research Notes in Math., V.5, (1975) Pitman, London.

[22] P. Kersten, I. Krasil'shchik, and A. Verbovetsky, Hamiltonian operators and $\ell^{*}$-coverings, J. Geom. Phys. 50 (2004), 273-302, arXiv: math/0304245.

[23] P. Kersten, I.S. Krasil'shchik, A.M. Verbovetsky, R. Vitolo: On integrable structures for a generalized Monge-Ampere equation, Theor. Math. Phys. 128, no. 2 (2012), 600-615.

[24] J.S. Krasil'shchik, A.M. Verbovetsky, R.F. Vitolo: The symbolic computation of integrability structures for PDEs, book, to appear in the Springer series "Texts and Monographs in Symbolic Computations" (2017).

[25] P.O. Lax, Hyperbolic systems of conservation laws, Comm. Pure Appl. Math. 10 (1957), 537-566.

[26] L. Manivel, E Mezzetti, On linear spaces of skew-symmetric matrices of constant rank, Manuscripta Math. 117, no. 3 (2005) 319-331.

[27] O.I. Mokhov, Symplectic and Poisson structures on loop spaces of smooth manifolds, and integrable systems, Russian Math. Surveys 53 No. 3 (1998) 515-622.

[28] F. Palatini, Sui sistemi lineari di complessi lineari di rette nello spazio a cinque dimensioni, Atti Ist. Veneto 602 (1900) 371-383.

[29] M.V. Pavlov, R.F. Vitolo, On the bi-Hamiltonian geometry of WDVV equations, Lett. Math. Phys. 105, no. 8 (2015) 1135-1163. 
[30] G.V. Potemin, On third-order Poisson brackets of differential geometry, Russ. Math. Surv. 52 (1997) 617-618.

[31] G.V. Potemin, Some aspects of differential geometry and algebraic geometry in the theory of solitons. PhD Thesis, Moscow, Moscow State University (1991) 99 pages.

[32] B.L. Rozdestvenskii and N.N. Janenko, Systems of quasilinear equations and their applications to gas dynamics, Translated from the second Russian edition by J. R. Schulenberger. Translations of Mathematical Monographs, 55. American Mathematical Society, Providence, RI, (1983) 676 pp.

[33] G. Saccomandi, R. Vitolo: On the Mathematical and Geometrical Structure of the Determining Equations for Shear Waves in Nonlinear Isotropic Incompressible Elastodynamics, J. Math. Phys. 55 (2014), 081502 .

[34] D. Serre, Systems of conservation laws. 1, 2. Cambridge University Press, Cambridge, 1999-2000.

[35] B. Sévennec, Géométrie des systèmes hyperboliques de lois de conservation, Mémoire (nouvelle série) N56, Supplément au Bulletin de la Société Mathématique de France, 122 (1994) 1-125.

[36] S.P. Tsarev, On Poisson brackets and one-dimensional Hamiltonian systems of hydrodynamic type, Soviet Math. Dokl. 31 (1985), 488-491.

[37] REDUCE, a computer algebra system; freely available at Sourceforge: http://reduce-algebra.sourceforge.net/

[38] R.F. Vitolo, CDE: a Reduce package for integrability of PDEs, software, user guide and examples freely available at http://gdeq.org. 